Portland State University

PDXScholar

\title{
Wearable Inertial Sensors Allow for Quantitative Assessment of Arthroscopic Skill in a Cadaveric Shoulder Model
}

Bryan C. Schreiner

Portland State University

Follow this and additional works at: https://pdxscholar.library.pdx.edu/honorstheses

\section{Let us know how access to this document benefits you.}

\section{Recommended Citation}

Schreiner, Bryan C., "Wearable Inertial Sensors Allow for Quantitative Assessment of Arthroscopic Skill in a Cadaveric Shoulder Model" (2017). University Honors Theses. Paper 418.

https://doi.org/10.15760/honors.414

This Thesis is brought to you for free and open access. It has been accepted for inclusion in University Honors Theses by an authorized administrator of PDXScholar. Please contact us if we can make this document more accessible: pdxscholar@pdx.edu. 


\title{
Wearable Inertial Sensors Allow for Quantitative Assessment of Arthroscopic Skill in a Cadaveric Shoulder Model
}

\author{
by \\ Bryan C. Schreiner \\ An undergraduate honors thesis submitted in partial fulfillment of the \\ requirements for the degree of \\ Bachelor of Science \\ in \\ University Honors \\ and \\ Biology \\ Thesis Adviser \\ Dr. Jacqueline Brady
}

Portland State University 


\section{Abstract:}

Surgical simulation training has been proposed to counter any erosion of surgical education due to limited operating room exposure. Current resident assessment is limited to subjective global rating scales as no standardized models exist to quantifiably assess the improvement in surgical proficiency in the operating room. The question addressed in this study was twofold: 1) Do motion patterns during shoulder arthroscopy correlate with level of expertise, as they do during knee arthroscopy? 2) Can improvement in shoulder arthroscopy technique performed on a cadaveric shoulder model be quantifiably measured? A novel model was developed to quantify the improvement in surgical proficiency by implementing Opal inertial sensors to quantify degrees of movement in four planes of motion while performing a diagnostic arthroscopy on a cadaveric shoulder. An average of individual surgeon joint positions was analyzed by taking the difference between pretest and posttest procedures $(\mathrm{p}<0.05)$. Four conclusions can be drawn from the results. Most compelling of the conclusions was the observed improvement in the camera hand, but not the probe hand. These preliminary results are promising for future research of the tested novel model to quantify the improvement in surgical proficiency. This research suggests that future experiments in this field of study could be improved by the implementation of a modified experimental design based on the results observed from these experimental trials. This research also suggests that the independent assessment of both the probe hand and camera hand is critical to the analysis of improvement in surgical proficiency. 


\section{Introduction:}

Surgical simulation training has been proposed as a means to counter any erosion of surgical resident education that might occur due to work hour constraints that limit the exposure of residents to the operating room. ${ }^{5}$ Virtual surgical simulation programs for the education of surgical residents were first proposed over 20 years ago as a method to gain exposure to "reallife" training necessary to provide an optimum level of patient care. ${ }^{16}$ Virtual surgical simulation programs can be defined as the surgical training of individuals utilizing prosthetic anatomical models or digital recreation of an procedure using a computer. Since then, there have been numerous studies that have attempted to quantify resident proficiency in both virtual reality and cadaveric models. ${ }^{1,3-5,8,13}$ Successful identification of ideal evaluation methods are necessary to best assess the technical performance for a given surgical training objective. ${ }^{12}$ To combat the problem of a lack of standardized assessment tool, subjective global rating scales (GRS) like The Arthroscopic Surgery Skill Evaluation tool (ASSET ${ }^{\mathrm{TM}}$ ) have been developed. ${ }^{11}$ Unfortunately a standardized objective method of evaluating resident performance in arthroscopy utilizing both virtual and cadaveric models has yet to be developed. Arthroscopy is defined as a minimally invasive surgical procedure that is performed on a joint using a camera called an arthroscope which is inserted into the joint to evaluate and treat many orthopaedic conditions.

A 2014 study of arthroscopic simulator training models found that practice on arthroscopic simulators improves the performance on arthroscopic simulators; however they were unable to determine whether skills learned on the simulation models correlate to improved proficiency in the operating room. ${ }^{7}$ Wearable inertial sensors worn on the surgeon's limbs and trunk of the torso have been shown to have the potential to overcome the limitations of current 
assessment tools by providing objective measures of performance and feedback that could be worn under real operating conditions. ${ }^{6,10}$ The significance of this finding was that using a wearable inertial sensor would allow the evaluation of surgical proficiency utilizing both virtual and real tissue by analyzing motion metrics. A 2008 study correlated motion analysis metrics, procedure times and GRS data to evaluate arthroscopic experience using software built into the VR simulator ${ }^{4}$, although the clinical applicability of VR simulator training has already been established with an additional study published in $2013 .^{9}$

The problem has been that there was no standardized method to quantifiably assess the improvement of surgical proficiency in the operating room on living tissue. Currently, the ability to evaluate arthroscopic skill of residents has been limited to subjective global rating scales. Therefore, based on the success of wearable inertial sensors to objectively measure proficiency, a novel model to assess performance of orthopaedic residents while performing an arthroscopic procedure referred to as a diagnostic shoulder arthroscopy on a cadaveric shoulder was implemented. Cadaveric shoulder tissue was used to most closely simulate real conditions expected in the operating room and the study was performed in the VirtuOHSU Surgical Simulation Center. Pilot data collected by OHSU faculty from a single day of testing on knee arthroscopy indicated that motion patterns, as detected by wearable inertial sensors, correlated well with level of expertise in arthroscopy. Level of expertise was determined such that the greater number of degrees of movement required to complete a procedure correlated to a lower level of experience. Using this novel model, the question addressed in this study was twofold: 1) Do motion patterns during shoulder arthroscopy correlate with level of expertise, as they do during knee arthroscopy? 2) Can improvement in shoulder arthroscopy technique performed on a 
cadaveric shoulder model be quantifiably measured? Improvement in shoulder arthroscopy will be defined later in the methods section.

The benefit of acquiring an objective measurement to assess surgical proficiency was that it would aid in identifying struggling residents early, allowing for appropriate remediation. In addition, it would potentially allow flexibility in the educational curriculum by identifying those residents who have obtained basic proficiency and who then may move on to more elective and advanced training. The intended audience of this study consists of hospital administrators and surgical faculty involved with the training of residents and undergraduate learners. The null hypotheses are that first, motion patterns detected using wearable inertial sensors worn on the limbs and trunk of the torso during shoulder arthroscopy would correlate with level of expertise, and second, improvement in shoulder arthroscopy technique performed on a cadaveric shoulder model can be quantifiably measured over a three-day period.

\section{Methods:}

To test the question, eight subjects performed a diagnostic arthroscopy on cadaveric shoulders. Cadaveric tissue was supplied by OHSU Body Donation program to the OHSU Orthopaedic Surgery Residency Arthroscopy Boot Camp, a one-week immersive program in shoulder and knee arthroscopy. The right and left fresh frozen cadaveric shoulders were randomly assigned to each station by the OHSU Body Donations staff. Post testing was performed on the same shoulder side as the pretest cadaveric shoulder. Lab space and cadaveric tissue were funded by the OHSU School of Medicine. Participants were divided into novices, four junior residents referred to as PGY3's and intermediates, four senior residents referred to as 
PGY4's. Arm movement data was collected using inertial measurement units (Opal'M Sensors, APDM® Portland, Oregon $)^{2}$ by securing two sensors to each upper extremity on the lateral arm and one sensor to the trunk on the sternum. No sensors were placed on the dorsal forearm or lumbar spine. See Appendix Figure 5 for visual depiction of placement of Opal sensors. Inertial motion of the tested orthopaedic residents was measured while performing a diagnostic shoulder arthroscopy in standard sequence: assessment of the superior labrum, inferior labrum, posterior labrum, biceps tendon, and rotator cuff tendons. Completion was determined by successful entry of both camera and probe instruments and safe performance of the standard assessment of the shoulder joint. Inertial motion was collected using the Opal sensors and compared between the $1^{\text {st }}$ day of the residents training during their pretest and their $3^{\text {rd }}$ day of training for the posttest. Three days was selected as this was the length of time established for this resident training on shoulder arthroscopy. Kinematics of the elbow and shoulder joints collected from the Opal sensors were calculated utilizing a proprietary algorithm by APDM (Portland, OR) to find degrees of movement in four planes motion using biomechanical modeling based on a sequence of links connected by joints.

Results were returned in degrees in the following four planes of motion: elbow pronation and supination, shoulder abduction and adduction, shoulder flexion and extension, and finally elbow flexion and extension. See Appendix Figure 6 for visual depiction of tested planes of motion. Range of motion was calculated as the difference between the $5^{\text {th }}$ and $95^{\text {th }}$ percentiles for each joint motion to standardize the results $(\mathrm{p}<0.05)$. Range of motion required to complete the procedure was calculated for each individual separated by the four different planes of movement by taking the difference of pretest and posttest results. A simple average was taken for each plane of motion to consolidate the results for each individual $(\mathrm{p}<0.05)$ and will be referred to in 
this research as group surgeon joint position. These calculations were performed such that a positive value indicates a decrease in degrees of joint angle required to complete the shoulder arthroscopy. Data was additionally separated by level of expertise, PGY3 and PGY4, to compare the distribution of joint position for novices, and intermediates.

For clarity in reporting results, a weighted average of each of the four planes of motion was calculated to express the trends of the data as a single value. Weight value of the average was established by previous research that had found a stronger correlation between quantity of motion and level of expertise such that shoulder ab-adduction and elbow pronation and supination were valued as more important than shoulder flexion and extension and elbow flexion and extension. ${ }^{15}$

In addition to analyzing change in degrees of motion, a percent change was also analyzed. The difference between pretest and posttest degrees of movement was taken. Percent difference was calculated as the quotient of the difference between the $5^{\text {th }}$ and $95^{\text {th }}$ percentiles divided by the pretest multiplied by 100 . A positive value indicates a decrease in the percent change of joint angle required to complete the shoulder arthroscopy.

Improvement in this study is defined as a decrease in range of motion. This is indicated by a positive value on the graphs such that the more positive, the more improvement that was observed. The reason improvement is defined as a decrease in range of motion is that previous research has correlated level of expertise with quantity of motion such that the less motion required to complete a procedure correlated to a higher level of skill. ${ }^{15}$

\section{Results:}


According to Table 1, all four planes of motion were found to yield inconsistent results with both improvement and lack of improvement. These results can be observed in Figure 1. It was also observed that regardless of the hand or cadaveric shoulder side there was both improvement and lack of improvement. The greatest improvement was recorded in the Shoulder Flexion/Extension plane with $19.6^{\circ}$ less than the pretest to complete the procedure with the left hand on the left cadaveric shoulder. The greatest lack of improvement was recorded in the Elbow Pronation/Supination plane with $27.3^{\circ}$ more than the pretest to complete the procedure with the right hand on the right cadaveric shoulder. The calculated weighted average found that improvement of the right hand on the left cadaveric shoulder and the left hand on the right cadaveric shoulder with $1.2^{\circ}$ and $4.1^{\circ}$ less than the pretest to complete the procedure respectively. It was also found that lack of improvement of the left hand on the left cadaveric shoulder and the right hand on the right cadaveric shoulder with $0.2^{\circ}$ and $16.1^{\circ}$ more than the pretest to complete the procedure respectively. These results can be observed in Figure 2.

According to Table 2, it was found that when both right and left cadaveric shoulders were considered together, the right hand consistently had a negative percent change with a weighted average of $-30.6 \%$. The left hand however was shown to have a $2.0 \%$ change for Shoulder Abduction/Adduction, however the other three planes of motion were all negative with a weighted average of $-14.9 \%$. When left and right cadaveric shoulders were considered separately, Elbow Pronation/Supination and Elbow Flexion/Extension were found to both have negative percent changes while Shoulder Abduction/Adduction and Shoulder Flexion/Extension was found to have inconsistent results with both positive and negative percent changes. The greatest positive percent change was recorded in the Shoulder Flexion/Extension plane at 30.1\% to complete the procedure with the left hand on the left cadaveric shoulder. The greatest negative 
percent change was recorded in the Shoulder Abduction/Adduction plane at $-66.8 \%$ to complete the procedure with the right hand on the right cadaveric shoulder. The calculated Weighted Average found a negative percent change regardless if the left or right cadaveric shoulder were considered together or separately. Results from the cadaveric left shoulder found a $-3.4 \%$ and $3.9 \%$ change for the left and right hand respectively. Results from the cadaveric right shoulder found a $-21.8 \%$ and $-46.6 \%$ change for the left and right hand respectively. Results discussed in Table 2 can be observed in Figure 3.

According to Figure 4, it was observed that PGY4's right hand had a greater negative percent change at $-51.6 \%$ in comparison the PGY3's percent change at $-9.6 \%$. Unlike the right hand, it was observed that PGY4's left hand had a smaller negative percent change at $-13.7 \%$ in comparison the PGY3's percent change at $-16.0 \%$. 


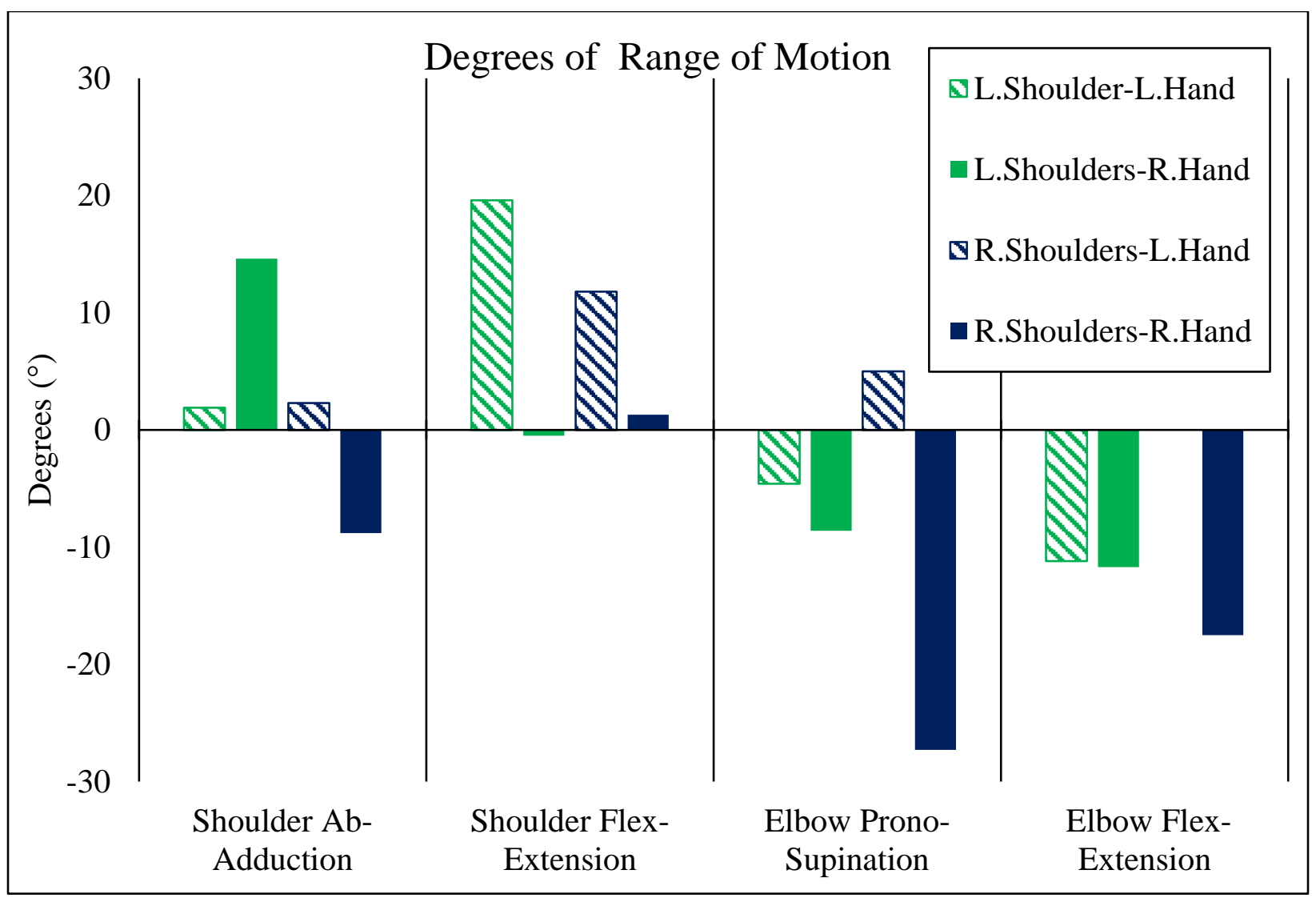

Figure 1. Group surgeon joint position representing the four tested planes of motion: shoulder abduction/adduction, shoulder flexion/extension, elbow pronation/supination, and elbow flexion/extension. Range of Motion calculated as the difference between the $5^{\text {th }}$ and $95^{\text {th }}$ percentiles for joint motion. All Measurements given as mean number of degrees $(\mathrm{p}<0.05)$. The difference between pretest and posttest degrees of movement was taken. A positive value indicates a decrease in degrees of joint angle required to complete the shoulder arthroscopy. Green results represent procedures performed on left cadaveric shoulders while blue represents procedures performed on right cadaveric shoulders. Striped results represent joint angles for the surgeon's left hand while solid results represent joint angles for the surgeon's right hand. 


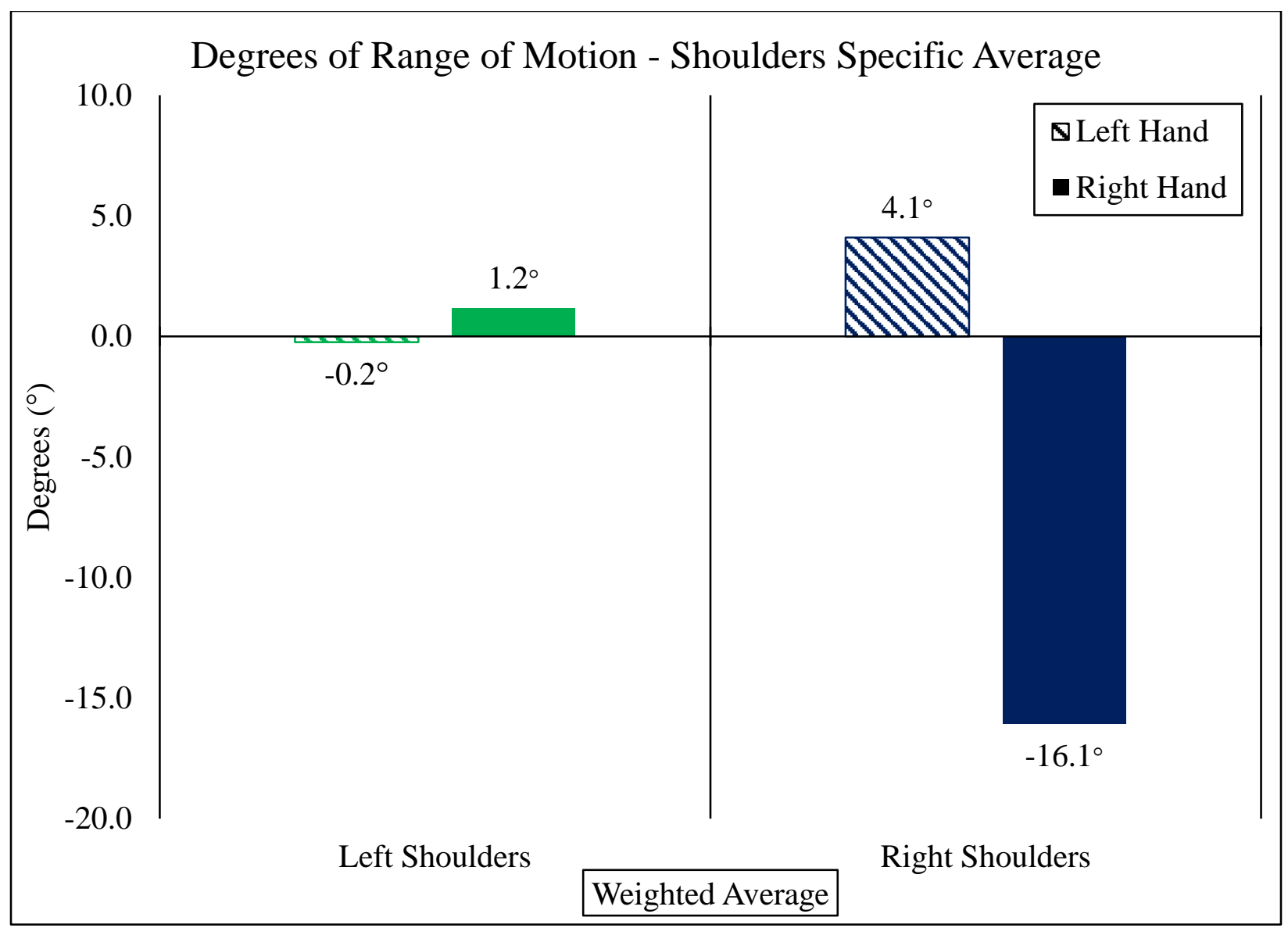

Figure 2. Group surgeon joint position represented as the weighted average of degrees of joint movement $(p<0.05)$. Weighted average was calculated to consolidate results of the four tested planes of motion. The weight value of the weighted average was based on previous research that showed a strong correlation between shoulder abduction/adduction and elbow pronation/supination and was therefore assigned a greater weight value. ${ }^{15}$ Results are divided by the left and right cadaveric shoulders as well as by the surgeons left and right hands such that green results represent procedures performed on left cadaveric shoulders while blue represents procedures performed on right cadaveric shoulders. Striped results represent joint angles for the surgeon's left hand while solid results represent joint angles for the surgeon's right hand. Range of Motion was calculated as the difference between the $5^{\text {th }}$ and $95^{\text {th }}$ percentiles for joint motion. All Measurements given as mean number of degrees $(\mathrm{p}<0.05)$. The difference between pretest and posttest degrees of movement was taken. A positive value indicates a decrease in degrees of joint angle required to complete the shoulder arthroscopy. 


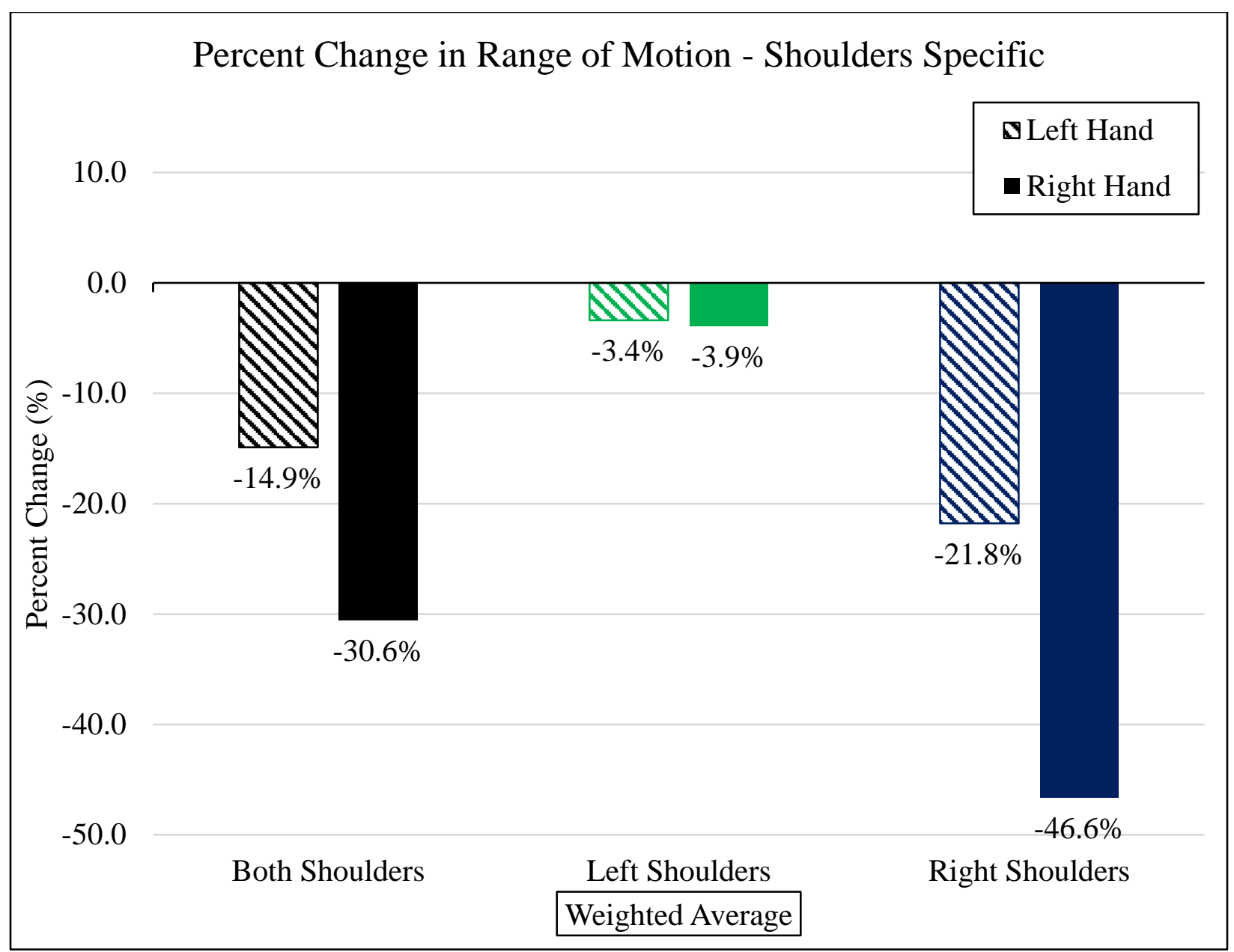

Figure 3. Group surgeon joint position represented as the weighted average of the percent change of joint movement $(\mathrm{p}<0.05)$. Weighted average was calculated to consolidate results of the four tested planes of motion. The weight value of the weighted average was based on previous research that showed a strong correlation between shoulder abduction/adduction and elbow pronation/supination and was therefore assigned a greater weight value. ${ }^{15}$ Results are divided by the left and right cadaveric shoulders as well as by the surgeons left and right hands such that green results represent procedures performed on left cadaveric shoulders while blue represents procedures performed on right cadaveric shoulders. Black represents an average of both left and right cadaveric shoulder results. Striped results represent joint angles for the surgeon's left hand while solid results represent joint angles for the surgeon's right hand. Range of Motion was calculated as the difference between the $5^{\text {th }}$ and $95^{\text {th }}$ percentiles for joint motion. All Measurements given as the percent change of degrees $(p<0.05)$. The difference between pretest and posttest degrees of movement was taken. Percent difference was calculated as the quotient of the difference divided by the pretest multiplied by 100. A positive value indicates an improvement in the percent change of joint angle required to complete the shoulder arthroscopy. 


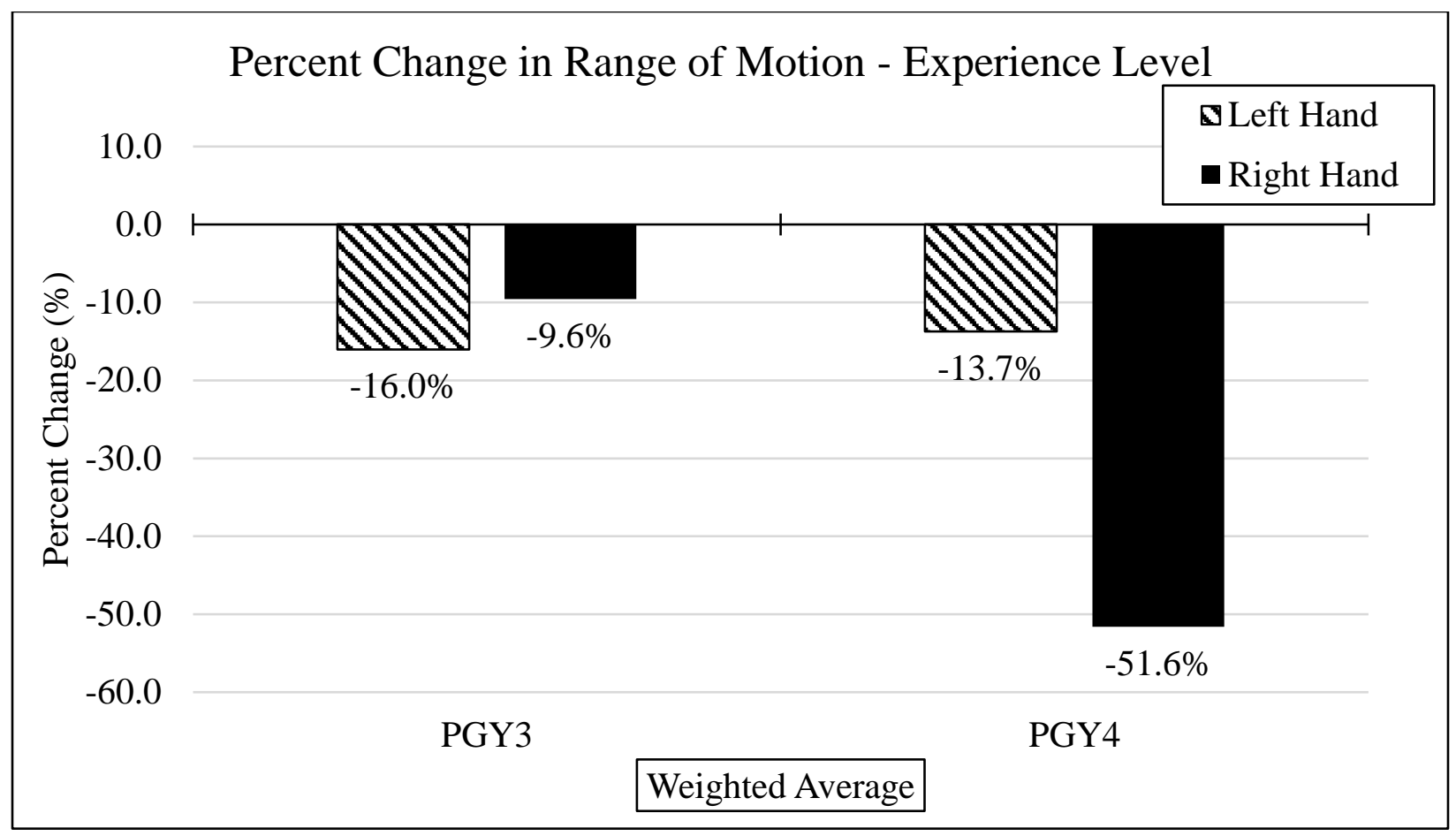

Figure 4. Group surgeon joint position represented as the weighted average of the percent change of joint movement $(\mathrm{p}<0.05)$. Weighted average was calculated to consolidate results of the four tested planes of motion. The weight value of the weighted average was based on previous research that showed a strong correlation between shoulder abduction/adduction and elbow pronation/supination and was therefore assigned a greater weight value. ${ }^{15}$ Results are divided by experience level and by the surgeon's left and right hands such PGY3 represent the more novice resident skill group in comparison to PGY4 resident skill group. Black represents an average of both left and right cadaveric shoulder results. Striped results represent joint angles for the surgeon's left hand while solid results represent joint angles for the surgeon's right hand. Range of Motion was calculated as the difference between the $5^{\text {th }}$ and $95^{\text {th }}$ percentiles for joint motion. All measurements given as the percent change of degrees $(p<0.05)$. The difference between pretest and posttest degrees of movement was taken. Percent difference was calculated as the quotient of the difference divided by the pretest multiplied by 100. A positive value indicates an improvement in the percent change of joint angle required to complete the shoulder arthroscopy. 


\begin{tabular}{|c|c|c|c|c|}
\hline \multicolumn{5}{|c|}{ Degrees of Range of Motion } \\
\hline & \multicolumn{2}{|c|}{ L. Shoulder } & \multicolumn{2}{|c|}{ R. Shoulder } \\
\hline & $\begin{array}{l}\text { L.Shoulder- } \\
\text { L.Hand }\end{array}$ & $\begin{array}{l}\text { L.Shoulders- } \\
\text { R.Hand }\end{array}$ & $\begin{array}{l}\text { R.Shoulders- } \\
\text { L.Hand }\end{array}$ & $\begin{array}{l}\text { R.Shoulders- } \\
\text { R.Hand }\end{array}$ \\
\hline $\begin{array}{l}\text { Shoulder Ab- } \\
\text { Adduction }\end{array}$ & $1.9^{\circ}$ & $14.6^{\circ}$ & $2.3^{\circ}$ & $-8.8^{\circ}$ \\
\hline $\begin{array}{l}\text { Shoulder Flex- } \\
\text { Extension }\end{array}$ & $19.6^{\circ}$ & $-0.5^{\circ}$ & $11.8^{\circ}$ & $1.3^{\circ}$ \\
\hline $\begin{array}{l}\text { Elbow Pro - } \\
\text { Supination }\end{array}$ & $-4.6^{\circ}$ & $-8.6^{\circ}$ & $5^{\circ}$ & $-27.3^{\circ}$ \\
\hline $\begin{array}{l}\text { Elbow Flex- } \\
\text { Extension }\end{array}$ & $-11.2^{\circ}$ & $-11.7^{\circ}$ & $0^{\circ}$ & $-17.5^{\circ}$ \\
\hline Weighted Average & $-0.2^{\circ}$ & $1.2^{\circ}$ & $4.1^{\circ}$ & $-16.1^{\circ}$ \\
\hline
\end{tabular}

Table 1. Group Range of Motion calculated as the average of the difference between the $5^{\text {th }}$ and $95^{\text {th }}$ percentiles for joint motion of each individual. All measurements given as number of degrees $(\mathrm{p}<0.05)$.

\begin{tabular}{|c|c|c|c|c|c|c|}
\hline \multicolumn{7}{|c|}{ Percent Change of Range of Motion } \\
\hline & \multicolumn{2}{|c|}{ Both Shoulders } & \multicolumn{2}{|c|}{ L. Shoulder } & \multicolumn{2}{|c|}{ R. Shoulder } \\
\hline & $\begin{array}{l}\text { Both Shoulders- } \\
\text { L.Hand }\end{array}$ & $\begin{array}{l}\text { Both } \\
\text { Shoulders- } \\
\text { R.Hand }\end{array}$ & $\begin{array}{l}\text { L.Shoulder } \\
\text {-L.Hand }\end{array}$ & $\begin{array}{l}\text { L.Shoulders } \\
\text {-R.Hand }\end{array}$ & $\begin{array}{c}\text { R.Shoulders } \\
\text {-L.Hand }\end{array}$ & $\begin{array}{l}\text { R.Shoulders } \\
\text {-R.Hand }\end{array}$ \\
\hline $\begin{array}{l}\text { Shoulder Ab- } \\
\text { Adduction }\end{array}$ & $2.0 \%$ & $-33.9 \%$ & $1.5 \%$ & $20.8 \%$ & $2.4 \%$ & $-66.8 \%$ \\
\hline $\begin{array}{l}\text { Shoulder } \\
\text { Flex- } \\
\text { Extension }\end{array}$ & $-3.7 \%$ & $-23.9 \%$ & $30.1 \%$ & $-12.8 \%$ & $-24.1 \%$ & $-30.6 \%$ \\
\hline $\begin{array}{l}\text { Elbow Pro- } \\
\text { Supination }\end{array}$ & $-33.1 \%$ & $-26.0 \%$ & $-6.0 \%$ & $-19.9 \%$ & $-49.4 \%$ & $-29.7 \%$ \\
\hline $\begin{array}{l}\text { Elbow Flex- } \\
\text { Extension }\end{array}$ & $-20.8 \%$ & $-42.0 \%$ & $-46.1 \%$ & $-29.7 \%$ & $-5.6 \%$ & $-49.4 \%$ \\
\hline $\begin{array}{l}\text { Weighted } \\
\text { Average }\end{array}$ & $-14.9 \%$ & $-30.6 \%$ & $-3.4 \%$ & $-3.9 \%$ & $-21.8 \%$ & -46.6 \\
\hline
\end{tabular}

Table 2. Group Range of Motion calculated as the average of the difference between the $5^{\text {th }}$ and $95^{\text {th }}$ percentiles for joint motion of each individual. All Measurements given as the percent change in number of degrees $(\mathrm{p}<0.05)$. 


\section{Discussion:}

Using a novel model, the question addressed in this study was twofold: 1) Do motion patterns during shoulder arthroscopy correlate with level of expertise, as they do during knee arthroscopy? 2) Can improvement in shoulder arthroscopy technique performed on a cadaveric shoulder model be quantifiably measured?

Based on the results, the null hypothesis was rejected and the alternative hypothesis was accepted for both hypotheses: First, motion patterns detected using wearable inertial sensors worn on the surgeon's limbs and trunk of the torso during shoulder arthroscopy did not correlate with level of expertise. Second, improvement in shoulder arthroscopy technique performed on a cadaveric shoulder model was not able to be quantifiably measured over a three-day period. Three observations can be drawn from the results. First, according to Figure 4 which depicts the percent change in degrees of motion vs expertise, it was found that results did not support the first hypothesis. There does not appear to be any correlation between motion patterns and level of expertise. It was expected that the PGY4's would have a greater positive value compared to the PGY3's; however, this was not observed. What is observed instead is nearly the opposite result in which the PGY4 group required more degrees of movement to complete the procedure compared to the PGY3 group on average. It is unknown why there was such a large discrepancy within the PGY4 group such that an additional 51.6\% degrees of motion were required to complete the procedure using their right hand. It was also observed that neither expertise group, PGY3 or PGY4, showed improvement with consistent negative percent changes.

Next, the second hypothesis was not consistently supported. According to Figure 2, there was improvement of the right hand on the procedure that used the cadaveric left shoulder as well as improvement of the left hand on the procedure that used the cadaveric right shoulder. 
Unfortunately, it was also observed that more motion was required by the left and right hand to complete the procedure on the cadaveric left and right shoulder respectively. This is a particularly interesting result as it indicates the different responsibilities that each hand has depending on if the left or right shoulder is being operated on. What this result shows is that the camera hand, but not the probe hand is constantly observed to improve. The hypothesis cannot be supported however, as it was expected that both hands would be observed to improve.

Finally, according to Figure 3 which depicts the percent change in degrees of motion vs the side of shoulder tissue tested on, it was found that the second hypothesis was not consistently supported. No improvement was observed as indicated by the consistent negative percent change. It was also observed that there was more motion required to complete the procedure on the right shoulder compared to the left shoulder as well as more motion required to complete the procedure with the right hand compared to the left hand.

It is likely that the data did not support either hypothesis because of limitations of the experimental design used for this preliminary research rather than a failure of the inertial Opal sensor to quantify movement. It is thought that these results are due to three experimental variables. First, it is likely that three days was an insufficient time to be able to quantify any improvement. It is possible that due to the degree of difficulty of the procedure, more time would be needed to see a quantifiable difference that accurately reflects surgeon proficiency. Second, it is likely that there was an insufficient range of experience to be able to see a difference between the tested level of experiences. PGY3's and PGY4's have only one year difference in procedural experience. Including a broader range of test subjects including medical students and faculty members to add depth to the results might reveal some quantifiable trends that relate level of expertise to degrees of motion required to complete a procedure. Finally, the shoulder tissue that 
was used was not pre-screened for tissue specific complications that could have increased the

procedural difficulty. This included the degree of arthritis and adhesions present in each joint. It is known that an increased presence of arthritis or adhesions increases the complexity of the shoulder arthroscopy likely leading to increased movement required over the course of the procedure to accommodate for the increased difficulty. If pretest and posttest cadaveric shoulders were equally complicated, the tested methodology would account for this as only the relative difference of the two results was analyzed in this experiment. Unfortunately, there is a higher degree of plasticity in the human tissue used in comparison to virtual or simulated models that tend to be more consistent. This means that it is very unlikely that human tissue will ever present with the same degree of complications. Human tissue was utilized in this experiment to determine in part if motion could be quantified under simulated clinical conditions that most closely represent conditions present when operating on a living human.

The weighted average applied to analyze results is not suspected to be a source of error in experimental design. The weight value chosen did not change the trends of the results, only the reported amplitude. This means that the weighted average did not change whether or not there was improvement or no improvement, only the amount value of either improvement or lack of improvement was altered. It is believed that the weighted average gives a more accurate value closer to the true single value result in comparison to a simple average based on previous research discussed in the methods. ${ }^{15}$

\section{Conclusion:}

Four primary conclusions can be drawn from the observed results. First, it is unclear as to why there was no correlation between improvement in motion patterns and level of expertise. 
Second, there was consistent improvement in the camera hand, but not the probe hand. This is likely due to the difference in procedural specific requirements of each hand. It is likely the technique of the camera hand was easier to improve compared to the technique required of the probe hand due to the relative increase in quantity of motion required by the probe hand to complete the procedure. This result is reassuring, however improvement in both hands was needed to support the hypothesis in this experiment. Third, there was more consistent movement required to complete the procedure on the right cadaveric shoulder than the left cadaveric shoulder. This is likely due to surgeon hand dominance and their individual procedural confidence. The role of each hand switches depending on what side of the body was being worked on such that the camera hand was always placed in the posterior portal, located on the back side of the shoulder. This suggests that more practice will be needed for this test group specifically on right shoulders to increase improvement. Finally, there was more consistent movement to complete the procedure with the right hand in comparison to the left hand. This suggests that surgeon hand dominance played a role in the procedure such that it is likely that the right hand was compensating for the left hand during the procedure. This finding may have been amplified by increased tissue specific complexity of the posttest shoulder in comparison to the pretest shoulder.

Future research possibilities will primarily address the three experimental variables believed to negatively influence the observed results in this experiment. First, increasing the number of days of practice between pretest and posttest procedures will likely increase the quantifiable difference between the two days. It is not currently known what the optimal time between initial pretest exposure and secondary post testing is to maximize any observed differences in improvement. Second, it is likely a broader range of experience would result in a 
greater ability to quantifiably analyze the difference between levels of experience. Finally, the preoperative screening of cadaveric shoulder tissue used for arthritis and adhesions would likely standardize the relative complexity of each shoulder joint. Postoperative surgeon surveys will not be used in future research to address this experimental weakness. Previous research has found that postoperative surveys used to quantify the surgeons perceived difficulty of the operation did not accurately reflect inertial motion measurements recorded in the same study. ${ }^{15}$

These results are promising for preliminary research of the tested novel model to quantify the improvement in surgical proficiency. However, it is thought that the data did not support either hypothesis because of the limitations of experimental design used for this preliminary research rather than a failure of the inertial Opal sensor to quantify movement. At the onset of experimentation, it was thought that the procedure was sufficient to achieve the measurements that we had sought out to quantify. This research suggests that the quality of results in future experiments in this field of study could be improved by the implementation of a modified experimental design based on the results observed from these experimental trials. This research also suggests that the independent assessment of both the probe hand and camera hand is critical to the analysis of improvement in surgical proficiency. Results collected from the procedures indicated that at most only an additional $20^{\circ}$ on average was needed to complete the procedures. Over the course of a long procedure this increase appears to be relatively small. The development of a model to assess surgical improvement is still important however. In a profession that deals with small details, the ability to quantify the tiny differences in surgical ability has large implications on the specific training of better future attending surgeons with the goal of improved patient care. 


\section{References:}

1. Angelo RL, Ryu RK, Pedowitz RA, Gallagher AG. The Bankart Performance Metrics Combined With a Cadaveric Shoulder Create a Precise and Accurate Assessment Tool for Measuring Surgeon Skill. Arthroscopy : the journal of arthroscopic \& related surgery : official publication of the Arthroscopy Association of North America and the International Arthroscopy Association. Sep 2015;31(9):1655-1670.

2. APDM. (2015). Research-Grade Wearable Sensors. Retrieved October 26, 2016, from http://www.apdm.com/wearable-sensors/

3. Cannon WD, Nicandri GT, Reinig K, Mevis H, Wittstein J. Evaluation of skill level between trainees and community orthopaedic surgeons using a virtual reality arthroscopic knee simulator. The Journal of bone and joint surgery. American volume. Apr 2 2014;96(7):e57.

4. Chang J, Banaszek DC, Gambrel J, Bardana D. Global Rating Scales and Motion Analysis Are Valid Proficiency Metrics in Virtual and Benchtop Knee Arthroscopy Simulators. Clinical orthopaedics and related research. Aug 182015.

5. Dunn JC, Belmont PJ, Lanzi J, et al. Arthroscopic Shoulder Surgical Simulation Training Curriculum: Transfer Reliability and Maintenance of Skill Over Time. Journal of surgical education. Aug 192015.

6. El-Gohary M, Holmstrom L, Huisinga J, King E, McNames J, Horak F. Upper limb joint angle tracking with inertial sensors. Conference proceedings : ... Annual International Conference of the IEEE Engineering in Medicine and Biology Society. IEEE Engineering in Medicine and Biology Society. Annual Conference. 2011;2011:5629-5632.

7. Frank RM, Erickson B, Frank JM, et al. Utility of modern arthroscopic simulator training models. Arthroscopy : the journal of arthroscopic \& related surgery : official publication of the Arthroscopy Association of North America and the International Arthroscopy Association. Jan 2014;30(1):121-133.

8. Gomoll AH, Pappas G, Forsythe B, Warner JJ. Individual skill progression on a virtual reality simulator for shoulder arthroscopy: a 3-year follow-up study. The American journal of sports medicine. Jun 2008;36(6):1139-1142.

9. Henn RF, 3rd, Shah N, Warner JJ, Gomoll AH. Shoulder arthroscopy simulator training improves shoulder arthroscopy performance in a cadaveric model. Arthroscopy : the journal of arthroscopic \& related surgery : official publication of the Arthroscopy Association of North America and the International Arthroscopy Association. Jun 2013;29(6):982-985.

10. Kirby, G. (2011). Create eLetters | The Journal of Bone \& Joint Surgery. Retrieved October 20, 2016, from http://jbjs.org/content/97/13/1119.eLetters

11. Koehler RJ, Amsdell S, Arendt EA, et al. The Arthroscopic Surgical Skill Evaluation Tool (ASSET). The American journal of sports medicine. Jun 2013;41(6):1229-1237.

12. Louridas, M., M.D. (2014). International assessment practices along the continuum of surgical training. Retrieved October 15, 2016, from http://www.sciencedirect.com.proxy.lib.pdx.edu/science/article/pii/S0002961016300812

13. Martin KD, Belmont PJ, Schoenfeld AJ, Todd M, Cameron KL, Owens BD. Arthroscopic basic task performance in shoulder simulator model correlates with similar task performance in cadavers. The Journal of bone and joint surgery. American volume. Nov 2 2011;93(21):e1271-1275.

14. Rebolledo, B. J. (2015, June). Arthroscopy Skills Development With a Surgical Simulator: A Comparative Study in Orthopaedic Surgery Residents. Retrieved October 19, 2016, from http://apps.webofknowledge.com.proxy.lib.pdx.edu/full_record.do?product=BIOSIS\&search_mode=Gener alSearch\&qid=8\&SID=3CRiHx7PKfcp2218U4Z\&page $=1 \&$ doc $=2$

15. Rose, M., MD, \& Curtze, C., PhD. (n.d.). Wearable Inertial Sensors Allow for Quantitative Assessment Of Arthroscopic Skill in a Cadaveric Knee Model.

16. Satava RM. Virtual reality surgical simulator. The first steps. Surgical endoscopy. May-Jun 1993;7(3):203205.

17. Shadmehr, R. (n.d.). Kinematics of a five-degree of freedom arm - Shadmehr lab. Retrieved November 10, 2016, from http://www.shadmehrlab.org/book/kinematics/kinematics.htm 


\section{Appendix:}

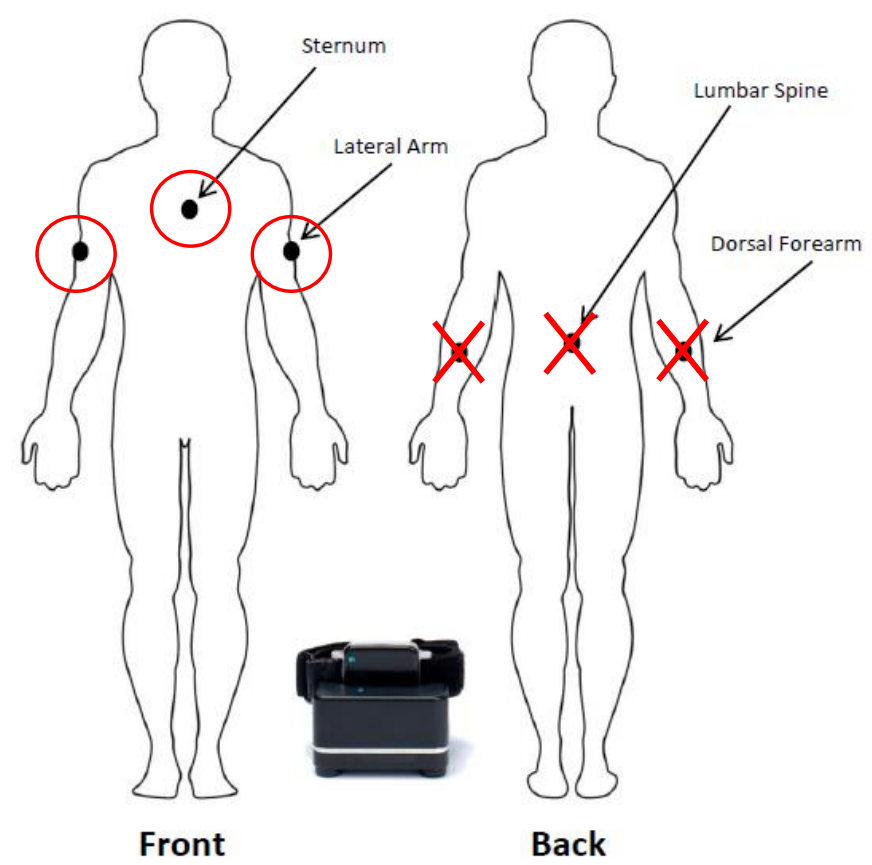

Figure 5. Opal ${ }^{\mathrm{TM}}$ Sensor and wireless docking station, APDM Inc. Portland, Oregon. In total, 3 sensors were placed on each subject for motion assessment (2 lateral arm, 1 sternum). Insignificant improvement in recoding accuracy with additional three sensors, 2 placed on the dorsal forearm, 1 on the lumbar spine. ${ }^{15}$

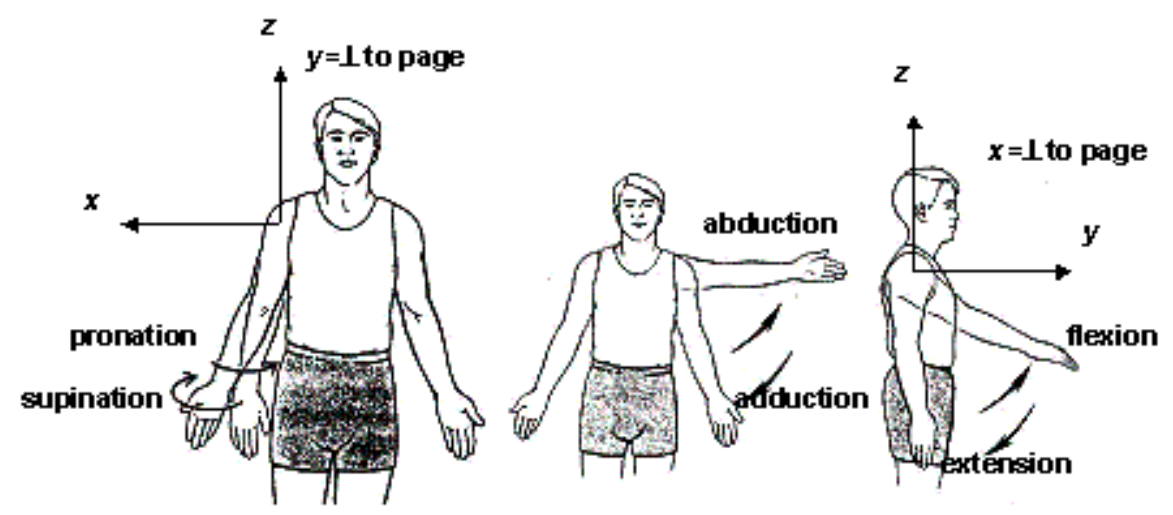

Figure 6. Roll, pitch, and yaw angles for the shoulder. Pronation and supination are rotations about the z-axis (yaw). Abduction and adduction are rotations about the y-axis (pitch). Flexion and extension are rotations about the $\mathrm{x}$-axis (roll). ${ }^{17}$ 